\title{
DIS3 mutations in multiple myeloma impact the transcriptional signature and clinical outcome
}

\section{Katia Todoerti, ${ }^{1,2^{*}}$ Domenica Ronchetti, ${ }^{2 *}$ Vanessa Favasuli, ${ }^{2}$ Francesco Maura, ${ }^{3}$ Fortunato Morabito, ${ }^{4,5}$ Niccolò Bolli, ${ }^{1,2}$ Elisa Taiana ${ }^{1,2 \#}$ and Antonino Neri ${ }^{1,2 \#}$}

${ }^{1}$ Hematology, Fondazione Cà Granda IRCCS Policlinico, Milan, Italy; ${ }^{2}$ Department of Oncology and Hemato-oncology, University of Milan, Milan, Italy; ${ }^{3}$ Myeloma Program, Sylvester Comprehensive Cancer Center, University of Miami, Miami, FL, USA;

${ }^{4}$ Biotechnology Research Unit, Aprigliano, A.O./ASP, Cosenza, Italy and ${ }^{5}$ Department of Hematology and Bone Marrow Transplant Unit, Augusta Victoria Hospital, Jerusalem, Israel.

*KT and DR contributed equally as co-first authors.

"ET and AN contributed equally as co-senior authors.

\section{ABSTRACT}

D IS3 gene mutations occur in roughly $10 \%$ of patients with multiple myeloma (MM); furthermore, DIS3 expression can be affected by monosomy 13 and del(13q), which occur in approximately $40 \%$ of MM cases. Despite several reports on the prevalence of DIS3 mutations, their contribution to the pathobiology of $\mathrm{MM}$ remains largely unknown. We took advantage of the large public CoMMpass dataset to investigate the spectrum of DIS3 mutations in MM and its impact on the transcriptome and clinical outcome. We found that the clinical relevance of DIS3 mutations strictly depended on the co-occurrence of del(13q). In particular, bi-allelic DIS3 lesions significantly affected progression-free survival, independently of other predictors of poor clinical outcome, while mono-allelic events mostly affected overall survival. As expected, DIS3 mutations affect the MM transcriptome involving cellular processes and signaling pathways associated with RNA metabolism, and the deregulation of a large number of long non-coding RNA, among which we identified five distinct transcripts as independent predictors of poorer overall survival and nine of worse progression-free survival, with two (AC015982.2 and AL445228.3) predicting both unfavorable outcomes. These findings strongly prompt further studies investigating the relevance of these long non-coding RNA in MM.

\section{Introduction}

Multiple myeloma (MM) is a hematologic malignancy that is still incurable despite the remarkable improvements in treatment and patients' care. ${ }^{1} \mathrm{MM}$ is characterized by a profound genomic instability involving ploidy, structural rearrangements, and a wide array of mutations affecting both putative oncogenes and tumor suppressor genes, such as KRAS, NRAS, TP53, BRAF, TRAF3, FAM46C, and DIS3..$^{2-7}$ These abnormalities are predicted to influence the biological and clinical behavior of the tumor and yet, despite a clear driver role, only a few carry prognostic value. ${ }^{8-10}$

Among mutated genes, DIS3 deserves great attention; this gene maps to 13q22.1 and encodes for a highly conserved ribonuclease indispensable for survival in vertebrates. ${ }^{11}$ DIS3 is a multidomain protein with two different catalytic activities: a 3'-5' exonucleolytic activity via the RNase II/R (RNB) domain and an endonucleolytic activity via the PilT N-terminal (PIN) domain at the N-terminus. ${ }^{11,12}$ DIS3 provides catalytic activity to the exosome, a multi-subunit complex involved in RNA degradation and metabolism, including mRNA quality control, gene expression regulation, and small RNA processing. ${ }^{12-15}$

DIS3 mutations and altered expression have been reported in roughly $10 \%$ of MM patients. ${ }^{6,16,17}$ Moreover, DIS3 expression can be affected by monosomy 13 and $\operatorname{del}(13 \mathrm{q})$, which occur in approximately $40 \%$ of MM patients. ${ }^{18,19}$ DIS3 muta-
Haematologica 2022

Volume 107(4):921-932

\section{Correspondence:}

ANTONINO NERI

antonino.neri@unimi.it

Received: January 14, 2021.

Accepted: April 22, 2021.

Pre-published: May 6, 2021

https://doi.org/10.3324/haematol.2021.278342

(C)2022 Ferrata Storti Foundation

Material published in Haematologica is covered by copyright. All rights are reserved to the Ferrata Storti Foundation. Use of published material is allowed under the following terms and conditions:

https://creativecommons.org/licenses/by-nc/4.0/legalcode. Copies of published material are allowed for personal or internal use. Sharing published material for non-commercial purposes is subject to the following conditions:

https://creativecommons.org/licenses/by-nc/4.0/legalcode, sect. 3. Reproducing and sharing published material for commercial purposes is not allowed without permission in writing from the publisher. 
tions are mainly located within the major ribonuclease domains of the protein, probably impairing DIS3 catalytic activity, as has been demonstrated to occur for some mutated residues. ${ }^{11}$ Recently, the analysis of a large cohort of MM patients enrolled in the Multiple Myeloma Research Foundation (MMRF) CoMMpass study pointed out that DIS3 mutations are almost exclusively missense, with no nonsense or frameshift mutations, copy neutral loss of heterozygosity, or bi-allelic deletions. In addition, a third of the mutations occur in three codons (D479, D488, and R780) within the RNB domain, almost never associated with del(13q); in contrast, the remaining two-thirds of DIS3 mutations are distributed across the various exons, almost always associated with del(13q)..$^{20}$

Despite the detailed overview of DIS3 mutations, their functional consequences on $\mathrm{MM}$ pathogenesis remain largely unknown, to the point that it is not clear whether DIS3 acts as an oncogene or a tumor suppressor gene. ${ }^{21,22} \mathrm{~A}$ crucial role for the DIS3 ribonuclease has been demonstrated in human MM cell lines, in which it promotes the maturation of the let-7 microRNA tumor suppressor family; indeed, through the reduction of mature let-7, DIS3 inactivation enhances the translation of let-7 targets such as MYC and RAS, leading to enhanced tumorigenesis. ${ }^{23}$ However, the extent to which this pathway is affected in MM patients harboring DIS3 mutations and how it may contribute to myelomagenesis need to be investigated further.

The clinical relevance of DIS3 mutations in MM was initially investigated by Weissbach et al:;2 despite the caution due to the small size of the cohort investigated, their data suggested that response to therapy was affected by DIS3 mutations depending on the presence of such mutations in minor rather than major subclones. Recently, the analysis of a larger cohort of MM cases at diagnosis revealed that DIS3 mutations were significantly associated with shorter event-free survival, showing an even worse outcome when in association with 13q deletion. However, overall survival (OS) was not affected. ${ }^{16}$ Notably, DIS3 mutations retained their significance for event-free survival in a multivariate analysis including $\mathrm{t}(4 ; 14)$ and the high-risk "double hit" group, i.e. patients with bi-allelic TP53 inactivation and amplification of $1 \mathrm{q} 21 .{ }^{10}$ However, it is becoming a well-established notion that, given the complexity of the genetic background in $\mathrm{MM}$, it is mandatory to assess DIS3 mutations in association with other oncogenic events, and their transcriptomic consequences, to establish their impact on MM prognosis. ${ }^{10,22,24}$

Based on these considerations, our study mined genomic and transcriptomic data from cases included in the MMRF CoMMpass dataset to dissect the impact of DIS3 mutations in the context of the MM genomic landscape in order to improve the definition of relevant clinical subsets. In addition, we investigated the transcriptomic profile related to DIS3 mutations to elucidate its role in myeloma cells and identify relevant pathways in MM pathobiology.

\section{Methods}

\section{Multi-omics data in the CoMMpass study}

Multi-omics data regarding bone marrow MM samples at baseline (BM_1) were freely accessible from the MMRF CoMMpass study (https://research.themmif.org) and retrieved from the Interim Analysis 12a (MMRF_CoMMpass_IA12a). Details about molecu- lar and clinical data of the CoMMpass cohort selected for the present study are described in the Online Supplementary Methods.

\section{Statistical and survival analyses}

Fisher exact test was applied to verify the association between genomic alterations in stratified $\mathrm{MM}$ cases. The two-tailed $P$-value was corrected using the Benjamini-Hochberg method, and adjusted $P$-values $<0.05$ were considered statistically significant. Survival analyses were performed using survival and survminer packages in $\mathrm{R}$ Bioconductor (version 3.5.1) as reported in the Online Supplementary Methods.

\section{Differential expression analysis of the CoMMpass cohort}

A global dataset from $655 \mathrm{MM}$ cases was stratified on the basis of DIS3 mutation RNA frequency and 56 DIS3-mutated cases with at least $20 \%$ RNA mutational load were considered in the differential expression analysis. Further steps of analysis are described in the Online Supplementary Methods. Principal component analysis was applied on differentially expressed (DE) annotated transcripts by means of the prcomp function in R. Volcano plots were used in $\mathrm{R}$ to represent significantly up- or down-regulated transcripts. The heatmap of the expression levels of the top $100 \mathrm{DE}$ annotated transcripts, according to the limma B statistics value, was created using dChip software. ${ }^{25}$

\section{Functional enrichment analysis on differentially expressed protein-coding genes}

Gene set enrichment analysis (GSEA) ${ }^{26}$ was performed on the pre-ranked DE protein-coding gene lists based on the fold change values by computing 1,000 permutations and using default analysis conditions. Further details about GSEA are provided in the Online Supplementary Methods.

\section{Long non-coding RNA expression validation}

We investigated long non-coding (lnc)RNA expression in a proprietary dataset that includes $43 \mathrm{MM}$ patients (Online Supplementary Table S1), upon written informed consent (Ethical Committee approval n. 575, 03/29/2018, Fondazione IRCCS Ca' Granda Ospedale Maggiore Policlinico). Further details about this analysis are reported in the Online Supplementary Methods.

\section{Results}

\section{Assessment of DIS3 mutations in myeloma patients}

We focused on a CoMMpass cohort of 930 bone marrow plasma cell samples from newly diagnosed MM patients for whom non-synonymous somatic mutation data, gained by whole exome sequencing, were available (Online Supplementary Table S2), identifying 103 DIS3 mutations in 94 of the 930 cases (Figure 1A, Online Supplementary Table S3). The variant allelic frequency ranged between 5.3\% and 100\% (mean: $48 \%$; median: $43 \%$ ). The majority of mutations were missense variants $(100 / 103)$ in the coding region (95/103) or within the region of the splice site (2/103), whereas in a minority of cases they consisted in start-lost (3/103). Three mutations were classified as splice acceptor or donor variants involving intronic sequences (3/103), all by means of SnpEff\&SnpSift tools (http://snpeff.sourceforge.net). Strikingly, DIS3 mutations mainly occurred in the active domains of the protein; in detail, 70/103 mutations fell within the RNB domain and ten in the PIN domain. With regard to the main mutational hotspots reported to occur within the RNB domain, R780 
A
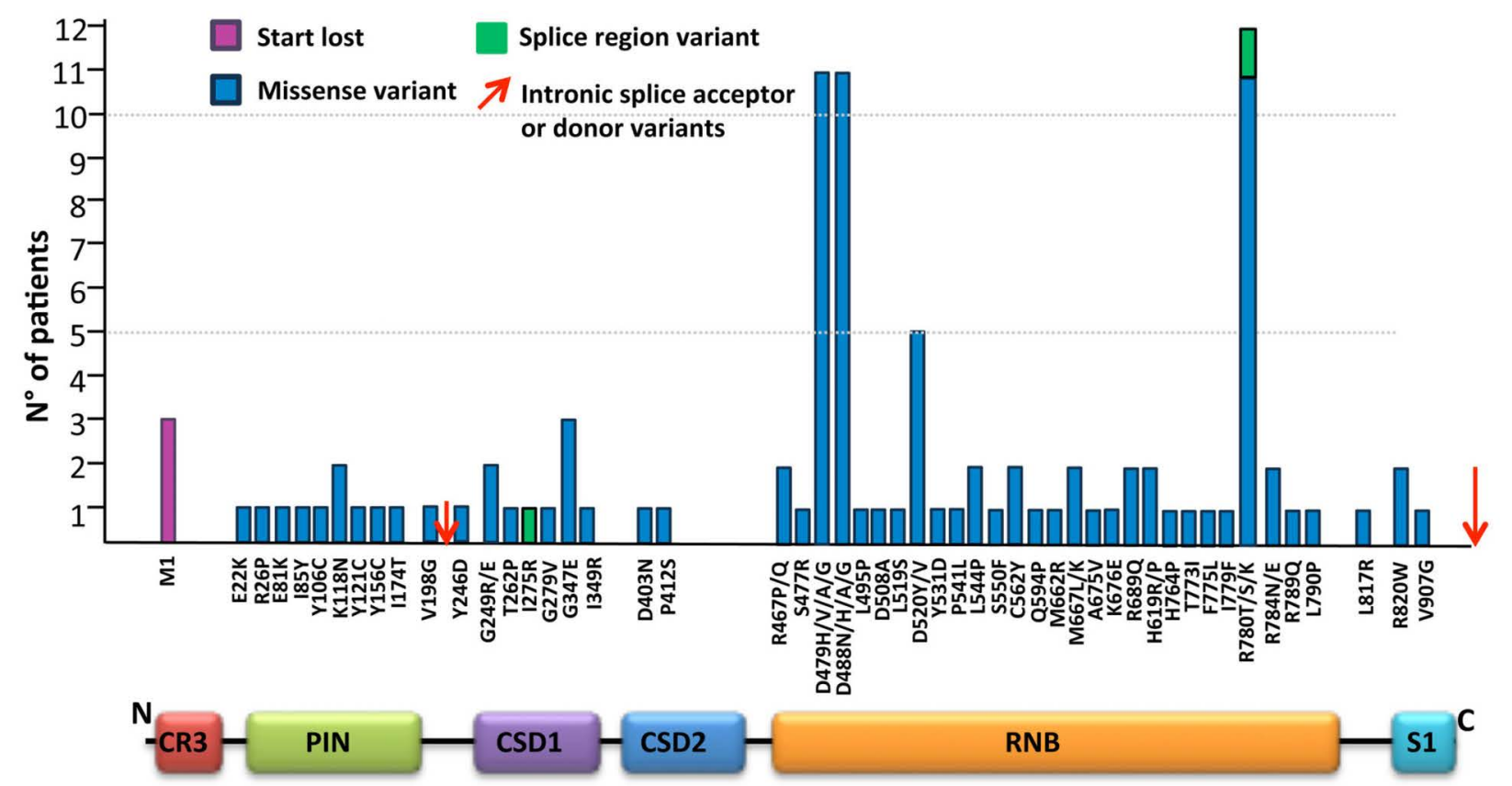

B

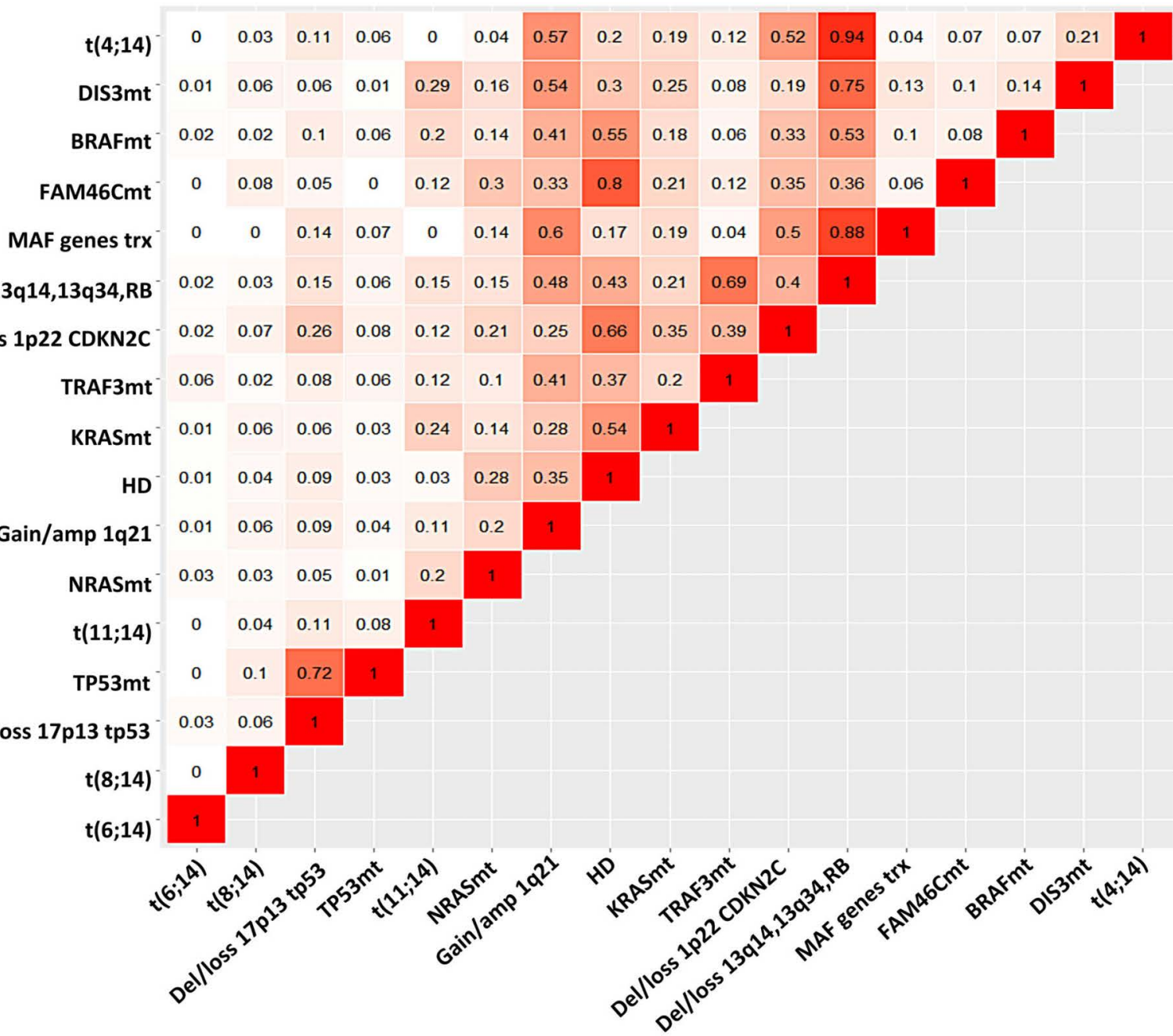

Figure 1. Distribution of DIS3 non-synonymous somatic variants in 94 patients with multiple myeloma of the CoMMpass cohort and the correlation of the variants with other genetic alterations. (A) Frequency of the 100 missense single nucleotide variants in DIS3 protein domains schematized below the histogram; the splice acceptor or donor variants occurring in intronic regions are indicated by red arrows. (B) Plot of co-occurrence of main copy number alterations, IGH translocations and non-synonymous somatic mutations in 651 cases of multiple myeloma in the CoMMpass dataset entirely profiled by whole exome sequencing and RNA-sequencing. 
Table 1. Cox regression univariate analysis in 630 patients with multiple myeloma for whom all data were available.

\begin{tabular}{|c|c|c|c|c|c|}
\hline \multirow[b]{2}{*}{ Variable } & \multirow[b]{2}{*}{ N (\%) } & \multicolumn{2}{|c|}{$\begin{array}{c}\text { OS } \\
\text { Univariate Cox Analysis }\end{array}$} & \multicolumn{2}{|c|}{$\begin{array}{c}\text { PFS } \\
\text { Univariate Cox Analysis }\end{array}$} \\
\hline & & adj.P-value & $\operatorname{HR}(95 \%$ Cl) & adj.P-value & HR ( $95 \%$ CI) \\
\hline ISS I & $223(35.4 \%)$ & 2.59E-06 & $0.26(0.15-0.43)$ & 2.00E-07 & $0.42(0.31-0.57)$ \\
\hline ISS II & $226(35.9 \%)$ & 7.13E-01 & $1.09(0.76-1.57)$ & $6.08 \mathrm{E}-01$ & $1.09(0.85-1.42)$ \\
\hline ISS III & $181(28.7 \%)$ & 1.92E-06 & $2.60(1.83-3.70)$ & 2.00E-07 & $2.08(1.62-2.68)$ \\
\hline $\operatorname{del}(13 q)$ & $281(44.6 \%)$ & $9.80 \mathrm{E}-02$ & $1.46(1.03-2.07)$ & 2.77E-01 & $1.21(0.94-1.55)$ \\
\hline DIS3mut & $17(2.7 \%)$ & $9.80 \mathrm{E}-02$ & $2.41(1.06-5.49)$ & 2.94E-01 & $1.67(0.83-3.39)$ \\
\hline $\operatorname{del}(13 q) / D I S 3 m u t$ & $298(47.3 \%)$ & 3.71E-02 & $1.63(1.14-2.32)$ & $1.52 \mathrm{E}-01$ & $1.27(0.99-1.63)$ \\
\hline $\operatorname{del}(13 q)+D I S 3 m u t$ & $50(7.9 \%)$ & $1.45 \mathrm{E}-01$ & $1.60(0.93-2.74)$ & 4.03E-02 & $1.71(1.15-2.54)$ \\
\hline TP53.alterations (del(17p)/TP53 or TP53mt) & $53(8.4 \%)$ & $9.42 \mathrm{E}-01$ & $0.96(0.50-1.84)$ & 7.63E-01 & $0.93(0.58-1.49)$ \\
\hline 1 q21 gain/amp & $207(32.9 \%)$ & $9.80 \mathrm{E}-02$ & $1.45(1.02-2.07)$ & $5.63 \mathrm{E}-02$ & $1.38(1.07-1.78)$ \\
\hline TP53.alterations + 1q21 gain/amp & $24(3.8 \%)$ & $1.60 \mathrm{E}-04$ & $3.64(2.00-6.62)$ & 4.80E-03 & $2.56(1.49-4.4)$ \\
\hline N-RAS mut & $142(22.5 \%)$ & $9.42 \mathrm{E}-01$ & $1.02(0.65-1.50)$ & $6.31 \mathrm{E}-01$ & $0.91(0.68-1.23)$ \\
\hline K-RAS mut & $155(24.6 \%)$ & 4.82E-01 & $1.18(0.80-1.74)$ & 5.79E-01 & $1.12(0.84-1.48)$ \\
\hline BRAF mut & $49(7.8 \%)$ & 2.64E-01 & $1.49(0.84-2.65)$ & 4.49E-01 & $1.26(0.81-1.96)$ \\
\hline RAS/BRAF mut & $310(49.2 \%)$ & $3.60 \mathrm{E}-01$ & $1.22(0.86-1.74)$ & $6.48 \mathrm{E}-01$ & $1.07(0.83-1.37)$ \\
\hline TRAF3 mut & $48(7.6 \%)$ & $1.10 \mathrm{E}-01$ & $0.39(0.14-1.05)$ & 6.48E-01 & $0.88(0.54-1.44)$ \\
\hline FAM46C mut & $64(10.2 \%)$ & 4.82E-01 & $1.27(0.73-2.21)$ & 4.49E-01 & $1.22(0.82-1.83)$ \\
\hline $\operatorname{del}(1 \mathrm{p}) / \mathrm{CDKN} 2 \mathrm{C}$ & $190(30.2 \%)$ & 7.43E-02 & $1.55(1.08-2.23)$ & $3.27 \mathrm{E}-01$ & $1.20(0.92-1.56)$ \\
\hline HD & $359(57.0 \%)$ & $9.80 \mathrm{E}-02$ & $0.70(0.50-0.99)$ & $6.60 \mathrm{E}-02$ & $0.75(0.58-0.96)$ \\
\hline WHSC1-FGFR3.trx & $86(13.7 \%)$ & $3.60 \mathrm{E}-01$ & $1.29(0.82-2.05)$ & $6.60 \mathrm{E}-02$ & $1.47(1.06-2.03)$ \\
\hline MAF.trx & $40(6.3 \%)$ & $1.03 \mathrm{E}-01$ & $1.78(1.00-3.16)$ & $3.83 \mathrm{E}-01$ & $1.32(0.83-2.09)$ \\
\hline MYC.trx & $26(4.1 \%)$ & $1.10 \mathrm{E}-01$ & $1.91(0.97-3.78)$ & $2.64 \mathrm{E}-01$ & $1.55(0.90-2.66)$ \\
\hline
\end{tabular}

Number $(\mathrm{N})$ and percentage (\%) of positive cases are indicated for each variable with the hazard ratio and $95 \%$ confidence interval. $P$ values that were statistically significant after Benjamini-Hochberg adjustment are reported in bold. OS: overall survival; PFS: progression-free survival; HR: hazard ratio; $95 \%$ CI: $95 \%$ confidence interval; ISS: International Staging System; del: deletion; mut: mutation; amp: amplification; HD: hyperdiploid; trx: translocation.

was involved in $12 \mathrm{MM}$ cases, whereas D488 and D479 residues were affected in $11 \mathrm{MM}$ samples each (Figure 1, Online Supplementary Table S3).

We evaluated the association of DIS3 mutations with the presence of the main IGH chromosomal translocations in $724 \mathrm{MM}$ cases (Online Supplementary Table S2). A significant co-occurrence was observed both with $t(4 ; 14)$ and MAF (MAF, MAFB, or MAFA) translocations $(P=0.0035)$. The association of DIS3 mutations with copy number alterations commonly found in MM disease was evaluated in $847 \mathrm{MM}$ cases, for which specific data were available by whole exome sequencing (Online Supplementary Table S2). As expected, a very significant association $(P=0.0003)$ was observed between DIS3 mutations and $\operatorname{del}(13 q)$ : specifically, 62 of 86 patients harboring a DIS3 mutation for whom data on copy number alterations were available showed a $13 q$ deletion (Online Supplementary Table S4). Next, we considered the distribution of $\operatorname{del}(13 q)$ across the different types of DIS3 mutations. Only nine of $29 \mathrm{MM}$ affected by the mutational hotspots also carried $\operatorname{del}(13 q)$; notably, the D479 hotspot was involved in five of these nine cases. In contrast, 50 of the 54 cases harboring not-hotspot DIS3 mutations were associated with $\operatorname{del}(13 q)(P<0.0001)$. Concerning the other main copy number alterations, a very significant association $(P=0.0020)$ was observed with 1 q21 gain/amplification (1q gain/amp), occurring in 45 of 86 DIS3-mutated patients, 38 of whom also carrying $\operatorname{del}(13 q)$. In contrast, a highly significant inverse correlation was found with the hyperdiploid condition $(P=0.0003)$ and the occurrence of $1 \mathrm{p} 22 / C D K N 2 C$ loss $(P=0.0205)$. Moreover, 17p13/TP53 deletions were present in only four of the 86 cases harboring DIS3 mutations (Online Supplementary Table S4).

We then looked at the co-occurrence of DIS3 mutations with the most frequently mutated genes in $\mathrm{MM}$, i.e., KRAS, NRAS, BRAF, FAM46C, TP53, and TRAF3 (Online Supplementary Table S2). A statistically significant association with DIS3 mutations was only observed for BRAF mutations $(P=0.0126)$ (Online Supplementary Table S4). Figure $1 \mathrm{~B}$ displays the global landscape of co-occurrence of DIS3 mutations with other main molecular lesions.

\section{Correlation of DIS3 mutations with clinical parameters}

We tested the clinical impact of DIS3 mutations in 930 MM cases with available PFS and OS data. At a median follow-up of 889 and 868 days for PFS and OS, respectively, a significantly lower survival rate for both OS (log-rank $P=0.039)$ and PFS (log-rank $P=0.021$ ) was observed in 94 DIS3-mutated cases compared to 836 DIS3 wild-type MM cases. Specifically, the median PFS was 800 days in DIS3-mutated cases versus 1176 days in wild-type MM cases (Figure 2A, B), whereas the OS evaluated at 3 years was $65 \%$ in DIS3-mutated cases versus $79 \%$ in the wildtype group.

As described above, DIS3 mutations co-occur significantly with $\operatorname{del}(13 q)$ and therefore with the loss of the second allele, a finding affecting several other genes in $\mathrm{MM}$. Such a result prompted us to stratify the cases in the CoMMpass series into four groups according to the absence of both mutated DIS3 and del(13q) (381 cases); the presence of only a DIS3 mutation (24 cases); the del(13q) alone (380 cases); or the occurrence of both a DIS3 muta- 
A

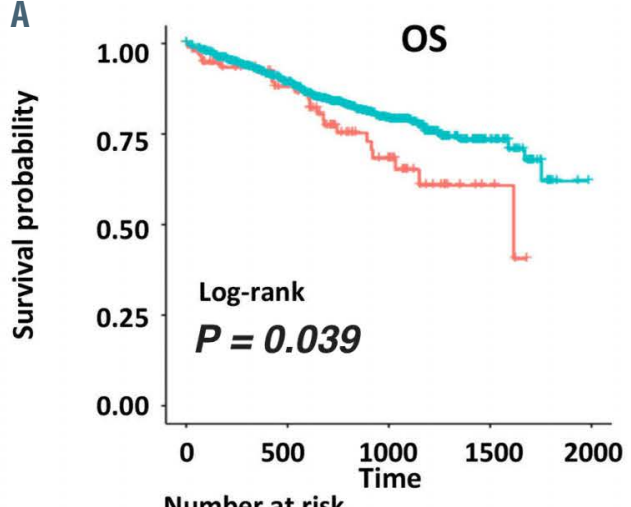

B

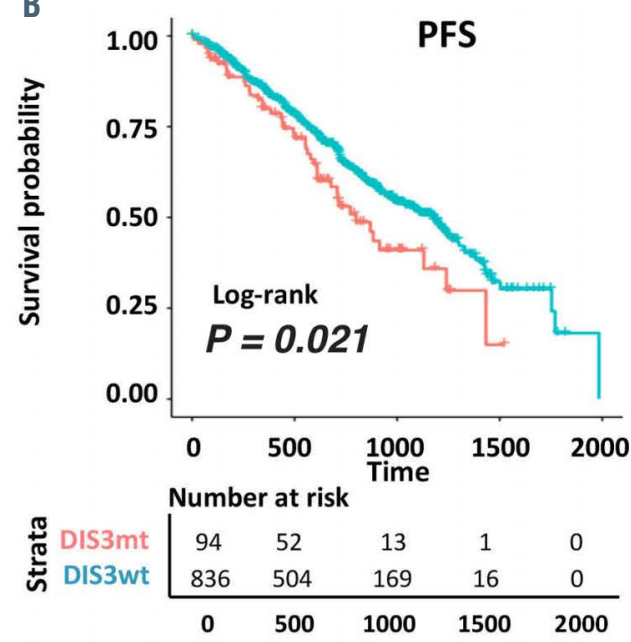

D

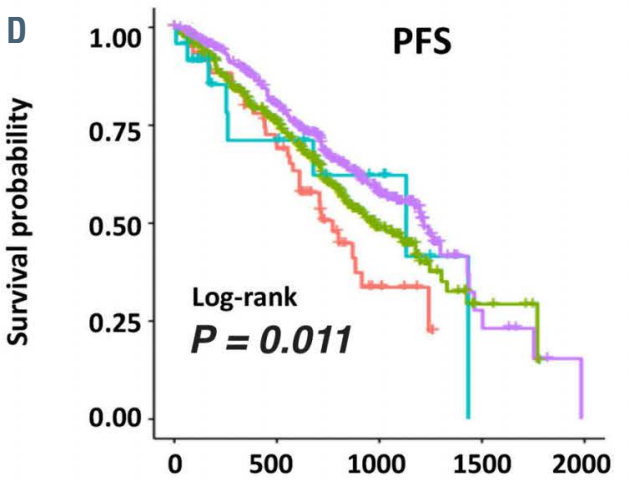

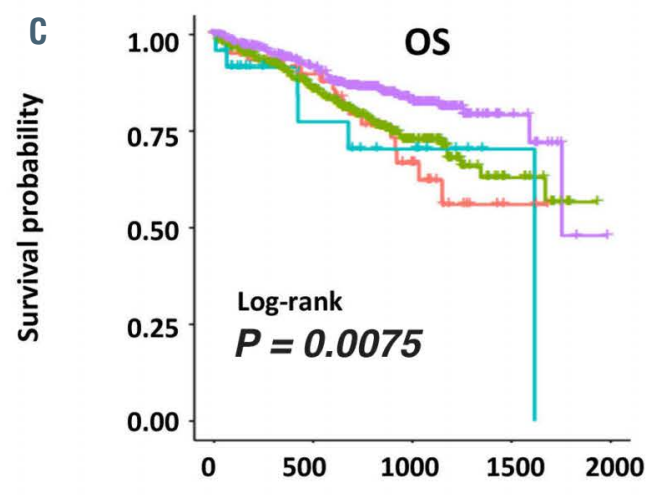

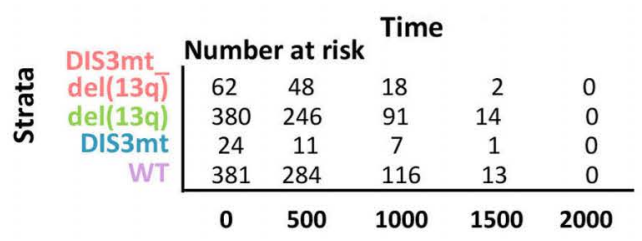

\begin{tabular}{|c|c|c|c|}
\hline OS & DIS3mt_del(13q) & \multicolumn{1}{c|}{$\operatorname{del}(\mathbf{1 3 q})$} & DIS3mt \\
\hline del(13q) & 0.686 & - & - \\
DIS3mt & 0.777 & 0.658 & - \\
WT & 0.044 & 0.022 & 0.089 \\
\hline
\end{tabular}

Figure 2. Impact of DIS3 mutations on clinical outcomes. (A, B) Kaplan-Meier survival curves of 930 patients with multiple myeloma (MM) stratified according to the occurrence of DIS3 mutations with respect to overall survival (A) and progression-free survival (B). (C, D) Kaplan-Meier survival curves of 847 patients stratified into four molecular groups according to the presence of DIS3 mutation and del(13q) as single or bi-allelic alterations, with respect to overall survival (C) and progression-free survival (D). Log-rank test $P$-value measuring the global difference between survival curves and numbers of samples at risk in each group across time are reported. Log-rank test $P$-values of pairwise comparisons are also reported in (C, D). Statistically significant adjusted $P$-values following Benjamini-Hochberg $(P<0.05)$ are in bold red. The median follow-up time for the overall survival analysis was 862 days (interquartile range, 594-1,092 days) while that for progression-free survival was 828 days (interquartile range, 535-1,094 days). OS: overall survival; PFS: progression-free survival; del: deletion; mt: mutated; WT: wild-type.

tion and del(13q) (62 cases). As depicted in Figure 2C, D, the presence of the bi-allelic alterations was associated with a poor prognosis in comparison to the wild-type condition, with respective 3 -year OS rates of $62 \%$ versus $82 \%$, and a median PFS of 772 days versus 1215 days.

Furthermore, we tested the prognostic impact on both PFS and OS of DIS3 mutations and $\operatorname{del}(13 q)$, as single or biallelic lesions, along with other parameters, in $630 \mathrm{MM}$ patients for whom all the information was available (Table 1). A significantly increased risk of death or progression was associated with International Staging System (ISS) stage III and the occurrence of $1 \mathrm{q}$ gain/amp in association with TP53 alterations. Moreover, a higher risk of having a shorter OS was also associated with DIS3 mutations or $\operatorname{del}(13 q)$ as single events (mono-allelic condition). In contrast, the bi-allelic condition was associated with a shorter PFS. In contrast, ISS stage I was associated with significantly shortened time to both death and progression (Table 1). Notably, all these features retained their independent prognostic power both for OS and PFS when tested in Cox regression multivariate analysis (Figure 3 ).

Based on such differential effects of mono- or bi-allelic DIS3 lesions, we re-analyzed the association of these events with the known MM oncogenic lesions. Regarding 
A

OS

Hazard ratio

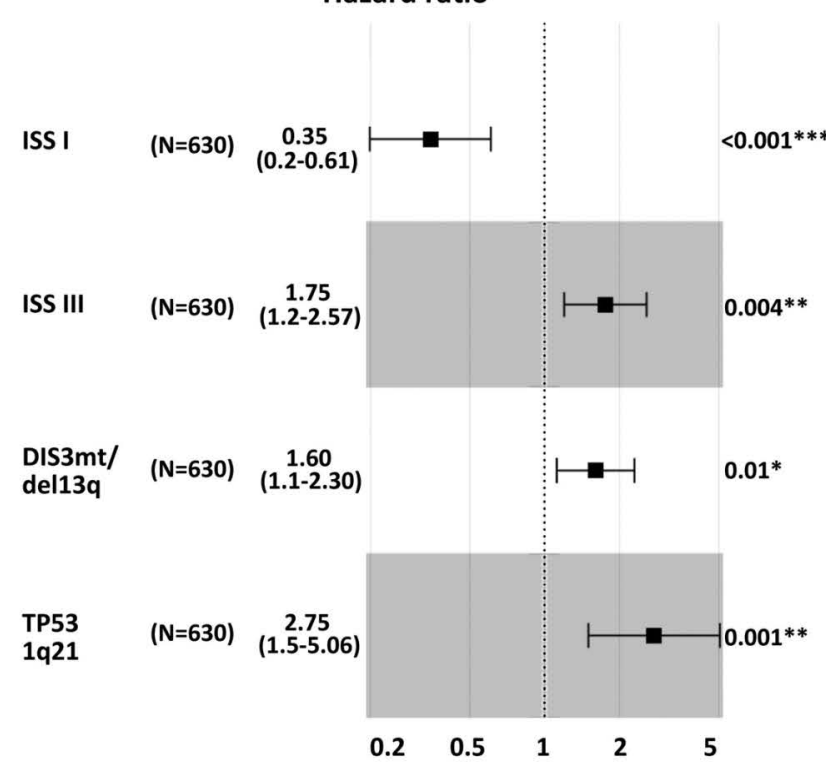

\# Events: 125; Global p-value (Log-Rank):1.756e-12

AIC: 1416.86; Concordance Index: 0.71
B

PFS

Hazard ratio

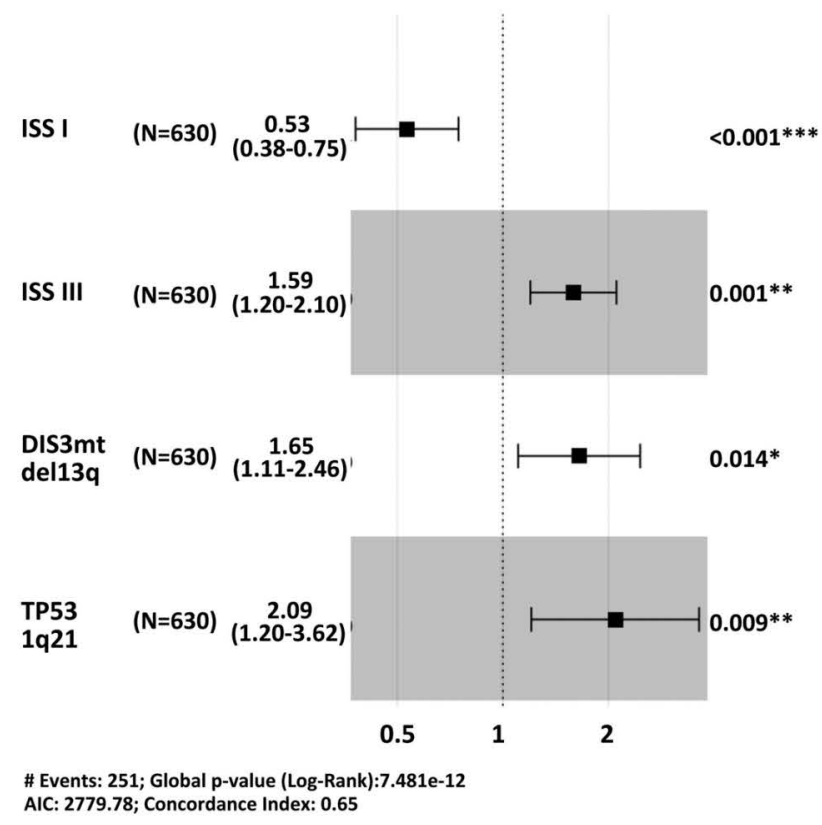

Figure 3. Impact of DIS3 mutations and other clinical/molecular variables on survival of patients with multiple myeloma. (A, B) Forest plots of Cox regression multivariate analyses considering all features with adjusted $P$-value $<0.05$ in univariate analysis with regards to overall survival (A) and progression-free survival (B), in 630 patients with multiple myeloma from the CoMMpass cohort for whom all considered data were available. The hazard ratio, $95 \%$ confidence interval and P-value are reported for each variable. A global log-rank P-value is reported for each analysis. OS: overall survival; PFS: progression-free survival; ISS: International Staging System.

the main IGH translocations, $\mathrm{t}(4 ; 14)$ was exclusively related to the bi-allelic condition $(P=0.0170)$. Furthermore, a higher prevalence of 1q gain/amp was observed in patients with bi-allelic DIS3 events (61\% vs. $29 \%$ ). Finally, a larger fraction of FAM46C mutated cases was evidenced in cases carrying only a DIS3 mutation (5.3\% vs. 3.1\%) (Online Supplementary Table S5).

Finally, we attempted to clarify further the prognostic relevance of DIS3 mutations in relation to well-established first-line regimens, as described in the CoMMpass dataset. In this respect, $930 \mathrm{MM}$ patients (94 of whom harboring DIS3 mutations) were grouped according to the type of therapy they had received: bortezomib/ immunomodulatory drug (IMID)-based, bortezomib-based, IMID-based, or carfilzomib-based (i.e., combining IMID/carfilzomib, bortezomib/IMID/carfilzomib, or single-agent carfilzomib schedules). Overall, carfilzomib-based regimens significantly improved both OS and PFS compared to all other types of combination therapies (Online Supplementary Figure S1). However, no significant difference in either OS or PFS was detected among DIS3-mutated cases irrespective of the specific therapy scheme (Online Supplementary Figure S2). These results suggest that DIS3 mutations could negatively affect carfilzomib-based therapies. However, further validation in a larger prospective cohort of patients is warranted.

\section{Transcriptional expression changes associated with DIS3 mutations and del(13q)}

To define the gene expression signatures and molecular pathways associated with DIS3 mutations, we focused on cases with expression of the mutation at meaningful levels, thus opting for a stringent cut-off of $20 \%$ on RNA mutational load (RNA_ALT_FREQ), chosen on the basis of analysis of the receiving operating characteristic curve (area under the curve, 98\%) according to DNA mutational level (variant allele frequency) (Online Supplementary Table S3, Online Supplementary Figure S3A). In further support of this cut-off value, we found a significant global correlation (Pearson correlation $\mathrm{r}=0.94$ ) between DNA and RNA mutational levels in those $56 \mathrm{MM}$ cases with $>20 \%$ DIS3 RNA mutational load, compared to the remaining $17 \mathrm{MM}$ cases with lower RNA somatic variant frequencies $(\mathrm{r}=0.30)$ (Online Supplementary Figure S3B, C). Furthermore, significantly shorter OS (log-rank $P=0.013$ ) and PFS (logrank $P=0.0013$ ) were observed in the $56 \mathrm{MM}$ cases with a $>20 \%$ DIS3 RNA mutational load compared to the 836 DIS3 wild-type cases. To note, no significant difference was appreciated between DIS3 sub-clonal mutations (i.e., $<20 \%$ DIS3 RNA mutational load) and wild-type cases (Online Supplementary Figure S4).

For the analysis, we therefore considered a total of 28,346 expressed transcripts and compared the expression profiles in 56 DIS3-mutated cases versus 582 DIS3 wildtype $\mathrm{MM}$ cases. A list of 7,167 DE transcripts, 6,564 of which annotated, was obtained at a low stringency cut-off (false discovery rate $<10 \%$ ). Among them, 3,464 proteincoding genes and 2,062 lncRNA resulted mostly upregulated $(79 \%)$ in DIS3-mutated patients compared to unmutated patients (Online Supplementary Table S6, Online Supplementary Figure S5). Principal component analyses based on the expression levels of the 6,564 DE annotated transcripts stratified according to the $\operatorname{del}(13 q)$ aberration confirmed the strong association between DIS3 mutations and del(13q) (Figure 4A). Furthermore, the heatmap of the top 100 most significantly upregulated transcripts, almost 
A

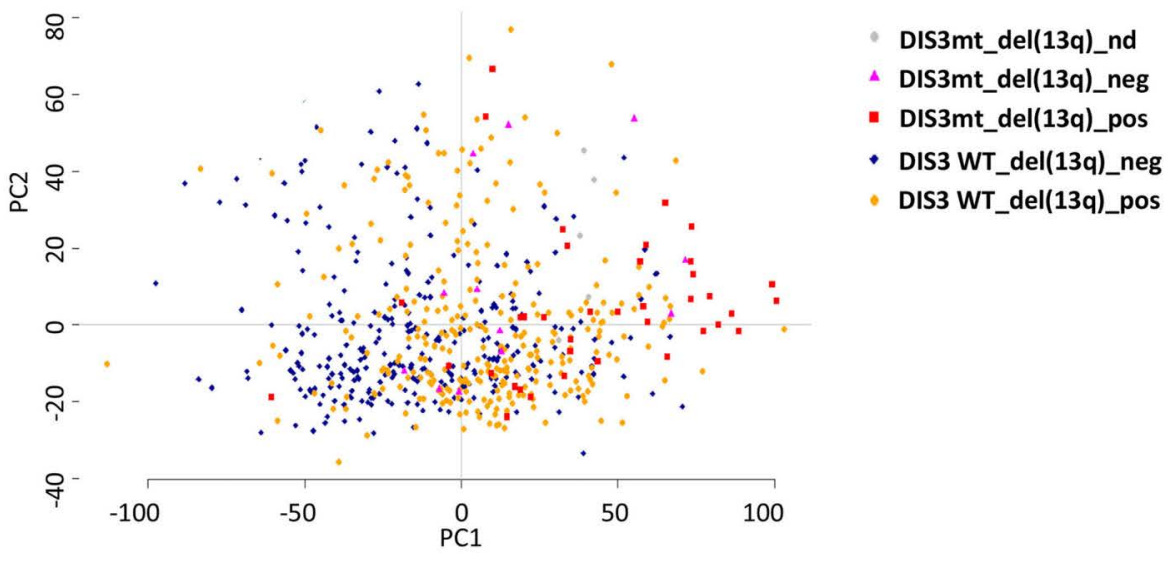

B

$\begin{array}{ccc}\text { DIS3mt }>20 \% & \text { DIS3 WT } & \text { DIS3 WT } \\ \text { del(13q) } & \text { del(13q)_pos } & \text { del(13q)_neg } \\ \text { nd pos neg } & \end{array}$

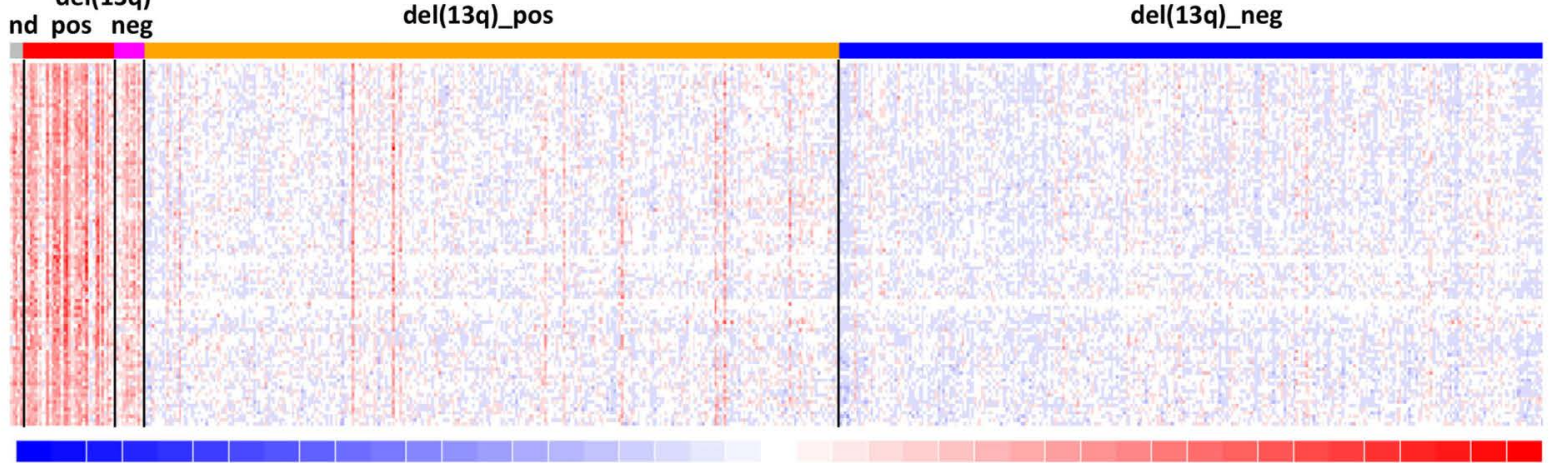

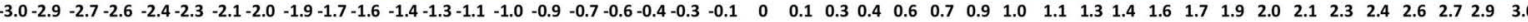

C

DIS3mt_del(13q)

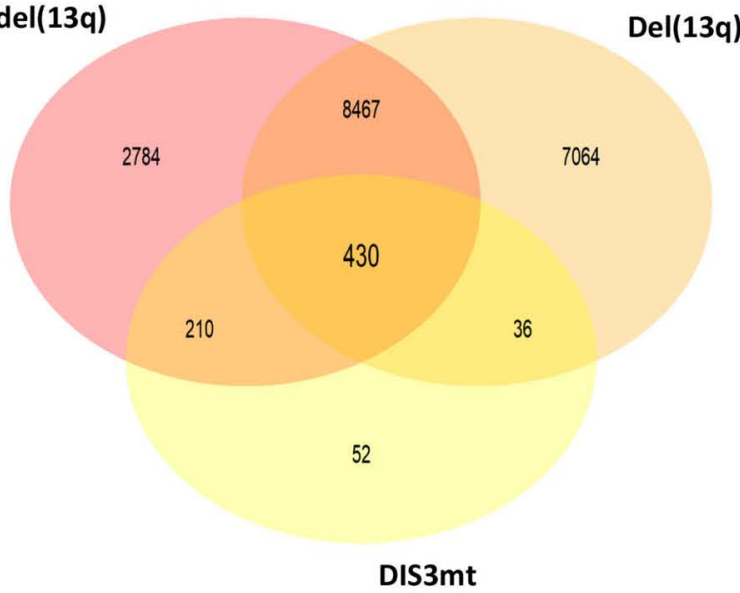

Figure 4. Transcriptional expression changes associated with DIS3 mutations and del(13q). (A) Principal component analyses of 6,564 differentially expressed transcripts in 56 cases of multiple myeloma (MM) with $>20 \%$ mutated DIS3 (DIS3mt) versus 582 DIS3 wild-type (DIS3 WT) cases. (B) Heatmap of the top 100 differentially expressed transcripts according to $B$ statistics value, in 56 DIS3 > 20\% mutated versus 582 DIS3 WT MM cases. The colored scaled bar represents standardized rows by subtracting the mean and dividing by the standard deviation. Samples in each group are further stratified according to the occurrence of $\operatorname{del}(13 q)$ aberration: 5 DIS3mt and not available (nd) for del(13q) MM cases, $38 \mathrm{MM}$ cases with bi-allelic alteration, $13 \mathrm{MM}$ carrying only DIS3mt, $289 \mathrm{MM}$ with del(13q) as a single lesion and 293 WT MM cases. (C) Venn diagram of differentially expressed transcript lists resulting from 13 MM with DIS3 mutation, $289 \mathrm{MM}$ with del(13q) as a single lesion, or 38 MM carrying bi-allelic alteration compared to 293 WT MM cases. all involving lncRNA (82\%), in the stratified MM samples revealed a stronger pattern of positive regulation in the biallelic condition with respect to DIS3 mutations or $\operatorname{del}(13 \mathrm{q})$ alone, compared to DIS3 wild-type MM cases (Figure 4B).

Based on these findings, we compared the global expression profiles associated with each lesion (13 cases with DIS3 mutations, 289 cases with $\operatorname{del}(13 q)$, and 38 cases with bi-allelic alteration) to the wild-type condition (293 cases). We found a shared set of 430 DE transcripts (of which 405 annotated) in all three comparisons, 305 of which were IncRNA, whereas 56 were classified as protein-coding genes (Figure 4C). Interestingly, 295 out of 405 transcripts showed a cumulative effect of deregulation across patients with mono-allelic and bi-allelic lesions (Online Supplementary Table S7).

Protein-coding genes: molecular pathways and gene sets modulated in association with DIS3 mutations

In order to define which molecular pathways could be modulated in relation to the occurrence of DIS3 mutations in MM, GSEA was performed on the list of DE proteincoding genes that were ranked based on fold change values (Online Supplementary Table S6).

The enrichment map on the top 100 GSEA gene sets based on gene ontology biological process terms revealed a complex network of connected functional modules 
Table 2. Summary information on the 12 long non-coding RNA significant in multivariate analysis.

\begin{tabular}{|c|c|c|c|c|c|c|c|}
\hline $\begin{array}{l}\text { Gene stable ID } \\
\text { Gene name * }\end{array}$ & Description \# & Chr. & Start (op) & End (bp) & Strand & $\begin{array}{l}\text { Neighbor } \\
\text { Gene } \\
\text { Name** }\end{array}$ & Pearson's correlation \\
\hline$\frac{\text { ENSG00000232519 }}{\text { AL353807.2 }}$ & $\begin{array}{l}\text { novel transcript, } \\
\text { AS to MSTO1 }\end{array}$ & lq22 & 155609776 & 155610380 & -1 & MST01 & $\mathrm{r}=0.38, P<6.08 \mathrm{E}-16$ \\
\hline$\frac{\text { ENSG00000235919 }}{\text { ASH1L-AS1 }}$ & $\begin{array}{c}\text { ASHIL AS RNA } 1 \\
\text { [Source:HGNC Symbol; } \\
\text { Acc:HGNC:44146] }\end{array}$ & lq22 & 155562042 & 155563944 & 1 & $\begin{array}{c}\text { ASH1L; } \\
\text { AL353807.4 }\end{array}$ & $\begin{array}{l}\mathrm{r}=0.34, P<6.08 \mathrm{E}-16 \\
\mathrm{r}=0.63, P<6.08 \mathrm{E}-16\end{array}$ \\
\hline$\frac{\text { ENSG00000271991 }}{\text { AC013400.1 }}$ & $\begin{array}{c}\text { novel transcript, } \\
\text { AS to TTC32 }\end{array}$ & $2 \mathrm{p} 24$ & 19902025 & 19902569 & 1 & TTC32 & $r=0.40, P<6.08 \mathrm{E}-16$ \\
\hline $\begin{array}{l}\text { ENSG00000271387 } \\
\text { AL445228.2 }\end{array}$ & $\begin{array}{l}\text { novel transcript, } \\
\text { AS to Clorf21 }\end{array}$ & lq25 & 184385753 & 184386704 & -1 & C1orf21 & $\mathrm{r}=0.69, P<6.08 \mathrm{E}-16$ \\
\hline $\begin{array}{l}\text { ENSG00000272606 } \\
\text { AC015982.2 }\end{array}$ & $\begin{array}{l}\text { novel transcript, } \\
\text { AS to PP4R3B }\end{array}$ & $2 p 16$ & 55617909 & 55618373 & 1 & PNPT1 & $\mathrm{r}=0.15, P=2.70 \mathrm{E}-05$ \\
\hline $\begin{array}{l}\text { ENSG00000236206 } \\
\text { AL356441.1 }\end{array}$ & $\begin{array}{l}\text { novel transcript, } \\
\text { sense to MGST3 }\end{array}$ & lq24 & 165598356 & 165624084 & 1 & MGST3 & $\mathrm{r}=0.28, P=8.93 \mathrm{E}-15$ \\
\hline $\begin{array}{l}\text { ENSG00000255647 } \\
\text { AC093510.1 }\end{array}$ & $\begin{array}{l}\text { novel transcript, } \\
\text { AS to CETN3 }\end{array}$ & $5 q 14$ & 90410000 & 90410669 & 1 & CETN3 & $\mathrm{r}=0.13, P=6.39 \mathrm{E}-04$ \\
\hline $\begin{array}{l}\text { ENSG00000259775 } \\
\text { AL138976.2 }\end{array}$ & $\begin{array}{l}\text { novel transcript, } \\
\text { AS to EIF5 }\end{array}$ & $14 q 32$ & 103331674 & 103332367 & -1 & EIF5 & $\mathrm{r}=0.30, P<6.08 \mathrm{E}-16$ \\
\hline $\begin{array}{l}\text { ENSG00000260236 } \\
\text { AC099778.1 }\end{array}$ & $\begin{array}{l}\text { novel transcript, } \\
\text { AS to PTPN23 }\end{array}$ & $3 \mathrm{p} 21$ & 47379089 & 47380999 & -1 & PTPN23 & $\mathrm{r}=0.11, P=4.42 \mathrm{E}-03$ \\
\hline $\begin{array}{l}\text { ENSG00000272426 } \\
\text { BX284668.6 }\end{array}$ & $\begin{array}{l}\text { novel transcript, } \\
\text { AS to CROCC }\end{array}$ & $1 p 36$ & 16904339 & 16904776 & -1 & $\begin{array}{c}\text { RNU1-2 } \\
\text { CROCC }\end{array}$ & $\begin{array}{l}\mathrm{r}=0.15, P=3.00 \mathrm{E}-05 \\
\mathrm{r}=0.18, P=1.36 \mathrm{E}-06\end{array}$ \\
\hline $\begin{array}{l}\text { ENSG00000272716 } \\
\text { AL121658.1 }\end{array}$ & $\begin{array}{l}\text { novel transcript, } \\
\text { AS to SLC30A6 }\end{array}$ & $2 \mathrm{p} 22$ & 32165046 & 32165757 & -1 & $\begin{array}{l}\text { SLC30A6 } \\
\text { SPAST }\end{array}$ & $\begin{array}{r}\mathrm{r}=0.07, P=8.94 \mathrm{E}-02 \\
\mathrm{r}=0.15, P=2.71 \mathrm{E}-05\end{array}$ \\
\hline $\begin{array}{l}\text { ENSG00000273355 } \\
\text { AP000894.4 }\end{array}$ & $\begin{array}{l}\text { novel transcript, } \\
\text { AS to YES1 }\end{array}$ & 18p11 & 813274 & 813756 & 1 & YES1 & $\mathrm{r}=0.16, P=1.93 \mathrm{E}-05$ \\
\hline
\end{tabular}

mainly concerning RNA and protein metabolism, cellcycle regulation, nucleosome organization, immune response, cell proliferation and apoptosis, cell adhesion and tissue development (Online Supplementary Figure S6). Furthermore, GSEA revealed a significant enrichment of transcriptional signatures some of which known to be distinctively associated with main $I G H$ translocations (Online Supplementary Table S8), likely in agreement with the cooccurrence of DIS3 mutations and $\mathrm{t}(4 ; 14)$ or MAF translocations (Online Supplementary Table S4), or in an opposite manner, with the hyperdiploid condition (Online Supplementary Tables S4 and S8). Therefore, with the aim of identifying DE transcripts more specifically related to DIS3 mutations, we investigated the transcriptional profiles in MM subgroups with a more homogeneous genetic background, each of them stratified according to the occurrence of DIS3 mutations: i.e., patients carrying $\mathrm{t}(4 ; 14)$ and $1 \mathrm{q}$ gain/amp; MAF translocations and $1 \mathrm{q}$ gain/amp; or hyperdiploid cases (Online Supplementary Figure $S 7 A$ ). Whereas no significant DE transcripts were identified in cases with both MAF translocations and $1 \mathrm{q}$ gain/amp (likely due to the limited number of cases), almost $90 \%$ of the DE transcripts from the other two comparisons overlapped with the original transcriptional signature (Online Supplementary Figure S7A). Overall, we identified 1,542 shared DE transcripts that could be likely considered as distinctively associated with DIS3 muta- tions; notably, these transcripts corresponded to the most significantly deregulated ones in the global DE list (Online Supplementary Table S6, Online Supplementary Figure S7B). Focusing on the shared 490 coding transcripts (Online Supplementary Table S6, Online Supplementary Figure S7C), GSEA identified a number of gene sets among those previously obtained from the global analysis; in particular, we confirmed downregulation of the oxidative phosphorylation gene set along with genes involved in RNA and amino acid metabolism and translation, and upregulation of the interferon signaling gene set (Online Supplementary Figure S8, Online Supplementary Table S8).

Finally, the shared DE transcript list associated with DIS3 mutations was particularly enriched in IncRNA (782/1542: $51 \%$ of all DE transcripts) (Online Supplementary Table S6, Online Supplementary Figure S7D), thus further supporting the notion of a distinctive and stronger impact of DIS3 mutations on the ncRNA transcriptome.

\section{Differential expression patterns of long non-coding RNA associated with DIS3 mutations}

LncRNA transcriptional patterns specifically associated with DIS3 mutations were further investigated. In detail, based on the pronounced heterogeneity of IncRNA and their lower expression levels as compared to coding transcripts, we applied a more stringent analysis on the 782 
shared DE IncRNA, and focused on the 50 most significant ones (false discovery rate $<1 \%$ ). All lncRNA were upregulated in DIS3-mutated cases compared to DIS3 wild-type cases and were mainly represented by IncRNA antisense to coding genes (80\%); the remaining cases included three
IncRNA sense to coding-genes, one long intergenic nonprotein coding RNA, two microRNA host genes and two divergent transcripts (Online Supplementary Table S9). Of note, the novel transcript Z93930.2 is located less than 100 bp antisense to the transcription factor XBP1, a well-estab-
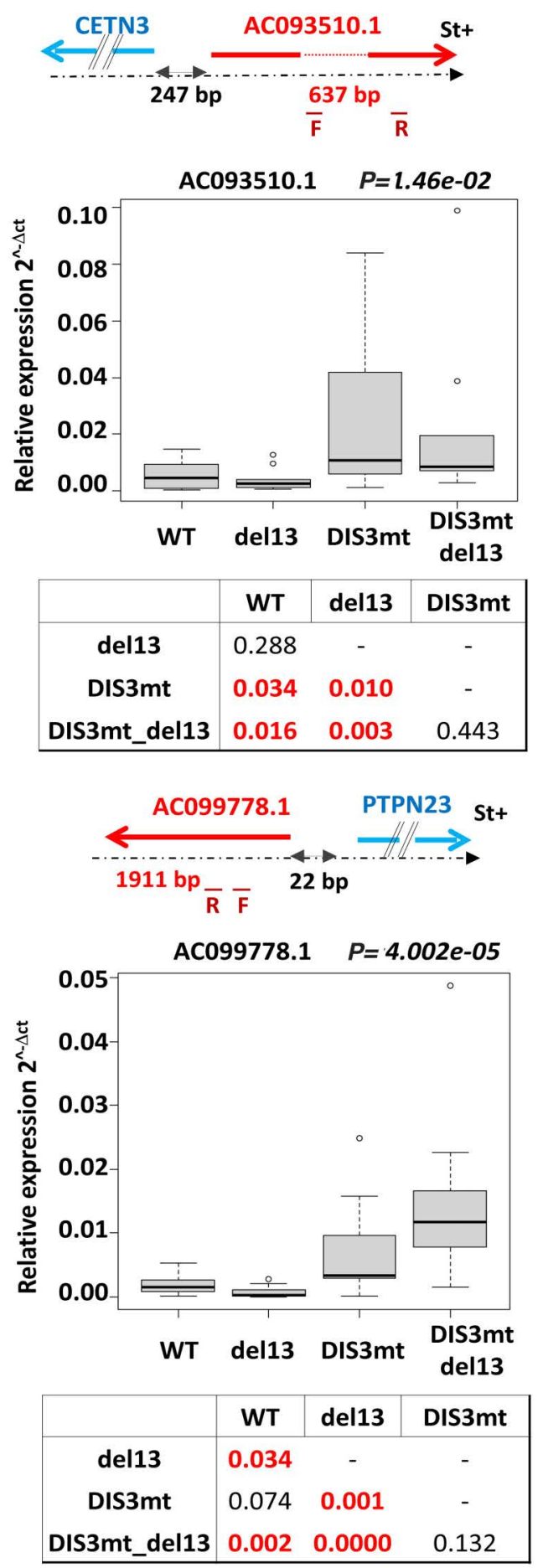
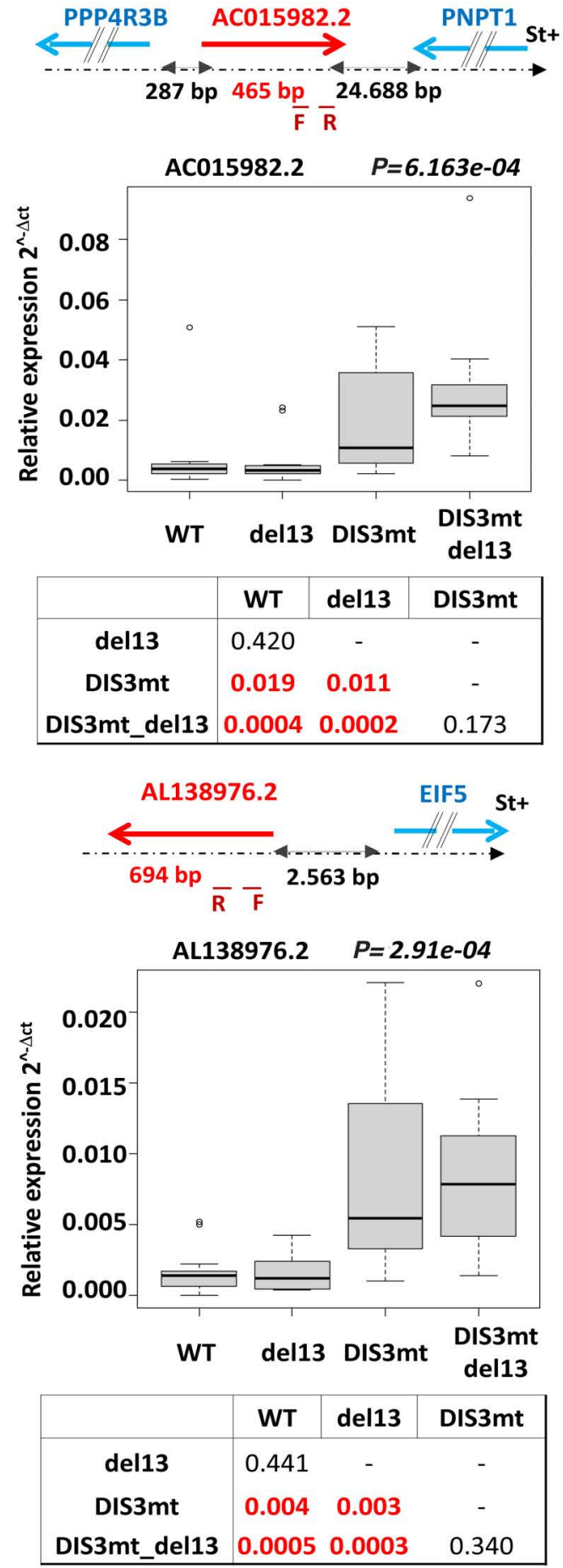

Figure 5. Long non-coding RNA with clinical relevance in patients with multiple myeloma. Scheme of the genomic region of the four long non-coding (Inc)RNA validated by means of quantitative reverse transcriptase polymerase chain reaction in 43 patients with newly diagnosed multiple myeloma including 13 with DIS3 wildtype (WT), 14 with DIS3 WT and del(13q), seven with DIS3 mutation (DIS3mt), and nine with DIS3mt and del(13q). Primer positions are indicated in red below each IncRNA. Differential expression was assessed by the Wilcoxon signed-rank test and statistically significant $P$-values $(<0.05)$ are reported above each boxplot. Dunn test. $P$-values for pairwise comparisons are reported in tables under each boxplot, with statistically significant $P$-values in bold red. 
lished regulator of $\mathrm{MM}$, known to be altered during the initiation and progression of $\mathrm{MM}^{27}$

Based on the recurrent evidence that the transcription of mRNA and IncRNA appears to be closely regulated, leading to a cis-regulatory relationship, ${ }^{28-30}$ we investigated the levels of expression of overlapping or nearby transcripts localized in close proximity to the $50 \operatorname{lncRNA}$ (in a window up to $65 \mathrm{~kb}$ ). We considered $81 \mathrm{mRNA}$-IncRNA pairs and analyzed the correlation between their expression levels across the entire dataset of $767 \mathrm{MM}$ cases profiled by RNA-sequencing in the CoMMpass cohort. A significant Pearson correlation ( $r>0.5, P<6.08 \times 10^{-16}$ ) was observed for nine lncRNA-gene pairs; among them, $A L 121672.3$ and MIRLET7BHG, both mapping at $22 \mathrm{q} 13$, showed a relevant correlation with each other $(\mathrm{r}=0.65)$ and with the PRR34 gene $(r=0.66$ and $r=0.73$, respectively) (Online Supplementary Table S10).

\section{Clinical relevance of long non-coding RNA}

Next, all 50 lncRNA were tested for their relationship to OS and PFS using Kaplan-Meier survival analysis on the $767 \mathrm{MM}$ cases with available RNA-sequencing and survival data. Groups with high versus low expression were determined according to the mean cut-off value for each lncRNA expression level across the entire dataset. Interestingly, higher levels of expression were associated with a poorer clinical outcome in terms of PFS for 35 out of all the 50 tested IncRNA. Furthermore, 15 of them showed an unfavorable prognosis in terms of OS (Online Supplementary Figure S9, Online Supplementary Table S11).

The clinical impact of the 35 IncRNA with poorer clinical outcome was further investigated by Cox regression univariate analysis. For $21 \operatorname{lncRNA}$, their higher expression level was associated with a significantly higher risk in PFS, and for five of them (AC015982.2, AL353807.2, AC013400.1, ASH1L-AS1, and AL445228.3) also in OS (Online Supplementary Table S12). Next, for all these 21 significant lncRNA, high and low lncRNA expression levels were tested in 630 cases, together with other clinically relevant characteristics, i.e. ISS stage I and III, the occurrence of 1q gain/amp in association with TP53 alterations, the presence of DIS 3 mutations or $\operatorname{del}(13 q)$ as single events for $\mathrm{OS}$, or in a bi-allelic condition for PFS. Notably, all the five IncRNA associated with a shorter OS retained their clinical impact when tested in Cox regression multivariate analysis. Two of them (AC015982.2 and AL445228.3) retained an independent significant prognostic power also for PFS; besides these two IncRNA, another seven (AC099778.1, AP000894.4, AL121658.1, AL356441.1, $B X 284668.6$, AC093510.1, AL138976.2) were found to be independent predictors of PFS at multivariate analysis (Table 2, Online Supplementary Table S13). Of note, the biallelic condition lost its independent clinical impact in all the multivariate analyses of the nine IncRNA significant for PFS (Online Supplementary Table S13).

Overall, from our analysis 12 IncRNA were predicted to have substantial clinical relevance (Table 2). Specifically, 11 of them code for novel transcripts, and four are antisense to known transcripts whose expression level, when detectable, was highly positively correlated (Table 2, Online Supplementary Table S10). Interestingly, we found four couples of IncRNA-coding genes (antisense or nearby) located on chromosome 1q with highly correlated expression (Table 2); these coding genes (MGST3, ASH1L, MSTO1, and C1orf21) were also significantly upregulated in DIS3-mutated samples as compared to unmutated ones (Online Supplementary Table S6). Finally, the expression of some (AC093510.1, AC015982.2, AL138976.2, and AC099778.1) of these IncRNA was validated by quantitative reverse transcriptase polymerase chain reaction in 43 newly diagnosed MM proprietary samples previously characterized for the presence of DIS3 mutations and for which material was available (Online Supplementary Table S1). In detail, we found that AC093510.1, AC015982.2, and AL138976.2 were significantly upregulated in MM with DIS3 mutations without del(13q) as compared to in DIS3 wild-type samples, whereas AC099778.1 was significantly upregulated only in the bi-allelic condition (Figure 5).

\section{Discussion}

We took advantage of the large publicly available CoMMpass dataset to investigate the type and frequency of DIS3 mutations in MM and their impact on the transcriptional signature and clinical outcome.

In agreement with previously reported data, ${ }^{6,16,17}$ we assessed that the frequency of DIS3 mutations in newly diagnosed $\mathrm{MM}$ is approximately $10 \%$; notably, DIS 3 mutations were associated with $\operatorname{del}(13 q)$ as bi-allelic events in $72 \%$ of cases. The majority of DIS3 mutations are missense. Together with their clustering at particular codons, the lack of truncating mutations is not typical of a tumorsuppressor gene and may suggest an oncogenic potential for DIS3. DIS3 mutations showed a clear pattern of cooccurrence with other molecular alterations, mainly chromosomal translocations. This phenomenon could be explained through the interaction between the RNA exosome and the protein activation-induced cytidine deaminase (AID) during the process of class switching and hypermutation in B cells; ${ }^{31}$ indeed, DIS3 mutations could indirectly, through disruption of an interaction with AID, cause mis-targeting of the somatic hypermutation process leading to chromosomal translocations. In particular, DIS3 mutations are associated with $\mathrm{t}(4 ; 14)$, this combination defining a poor prognostic MM subgroup. ${ }^{16}$ However, our analyses pointed out that $t(4 ; 14)$ occurred statistically significantly in patients with bi-allelic lesions, in agreement with the very frequent association between $t(4 ; 14)$ and $\operatorname{del}(13 q)$ alterations. Notably, the oncogenic events that most frequently co-occurred with DIS3 mutations were $\operatorname{del}(13 q)$, 1q gains, t(4;14) and MAF translocations. Overall, this spectrum of molecular lesions suggests a functional constraint of cooperating oncogenic events in MM, with a selection of later lesions being restricted by the ones appearing first in the transformed cell. With regard to this, DIS3 mutations were found to be both clonal in some patients and subclonal in others, meaning they might function sometimes as early and sometimes as late hits. ${ }^{7,22}$

Our study also pointed out that the clinical relevance of DIS3 mutations depended strictly on the co-occurrence of del(13q). In detail, we established that the bi-allelic lesions significantly affected PFS, whereas the mono-allelic condition predicted worse OS. Notably, these alterations remained valuable independent predictors even when tested in combination with the clinical and poor prognosis molecular variables used to foresee clinical outcome. The differential impact of the bi-allelic or mono-allelic lesions on MM outcome could be related to the fact that our data highlighted two patterns of DIS3 lesions: one in which 
$\operatorname{del}(13 q)$ co-exists with non-hotspot DIS3 mutations, and a second in which hotspot DIS3 mutations rarely show loss of heterozygosity through bi-allelic events. Again, this is a rather intriguing pattern that may suggest either haploinsufficiency for some mutations and not others, or a different function with some mutations showing loss and others showing gain of function. This might also explain the different clinical consequences on PFS and OS observed with the two genetic statuses. Indeed, previous reports showed how different types of DIS3 mutations could lead to diverse biological effects either by impairing exosome function through reduced/modified DIS3 activity $^{11}$ or through a dominant-negative effect exerted by mutated DIS3 on the other catalytic subunit, Rrp6, acting in the exosome complex. ${ }^{32}$

DIS3 is a key component of the multisubunit RNA exosome complex in eukaryotic cells involved in the processing, quality control and degradation of virtually all classes of RNA. Our study further supports and extends the notion that DIS3 mutations affect the transcriptome, showing a stronger impact on noncoding RNA species, mainly lncRNA. Indeed, we found that approximately half of the DE transcripts predicted to be specifically related to the presence of DIS3 mutations are represented by novel, largely uncharacterized lncRNA. Among them, we highlighted 12 lncRNA, five of which are independent predictors of poorer OS and nine of worse PFS, with two of them (AC015982.2 and AL445228.3) predicting both. Moreover, the clinical impact of the nine lncRNA predicting inferior PFS at higher expression levels was independent of the genetic status of DIS3. The effect of these 12 IncRNA on the pathobiology of MM disease remains to be fully elucidated; indeed, they are all novel transcripts, none of them currently reported as being associated with cancer. Interestingly, some of them showed a strict correlation in terms of expression levels with the corresponding nearby genes, thus suggesting a cis-regulatory relationship between the paired transcripts. Although these findings need to be investigated further, this could be the case for genes involved in fundamental molecular pathways, such as MGST3, involved in the production of leukotrienes and prostaglandin E, which are important mediators of inflammation; ${ }^{33}$ ASH1L, a methyltransferase already known to be involved in cancer; ${ }^{34}$ and MSTO1, important for mitochondrial fusion and intracellular distribution. ${ }^{35}$ Along with these findings, the patterns observed in the context of the DE coding transcripts associated with DIS3 mutations are also interesting. Indeed, we found downregulation of gene sets related to oxidative phosphorylation, metabolism of RNA or amino acids, and translation, and contrariwise, upregulation of interferon signaling. These transcriptomic changes are in line with the functional role of DIS3 in RNA metabolisms ${ }^{36,37}$ and in agreement with what has been described for yeasts in which mutations have been extensively investigated. ${ }^{38}$ In particular, the predicted enhanced interferon activity may represent a response to the accumulation of RNA substrates in cells following a deficiency of DIS3. ${ }^{39}$

Overall, our comprehensive evaluation of the clinical and transcriptional consequences of DIS3 mutations/deficiency in MM strongly indicates that they may play an important role in the mechanisms of MM transformation and progression. Our results may provide important insights for functional studies in order to better understand such mechanisms in MM.

\section{Disclosures}

No conflicts of interest to disclose.

\section{Contributions}

$K T$ designed the study, collected and analyzed data and wrote the paper; DR designed the study, analyzed data and wrote the paper; VF, FM and FM analyzed data; NB wrote the paper; ET and AN designed the study, supervised the study and wrote the manuscript

\section{Funding}

This work was financially supported by grants from the Associazione Italiana Ricerca sul Cancro (AIRC) to AN (IG16722 and IG24365). NB is funded by the European Research Council under the European Union's Horizon 2020 research and innovation program (grant agreement $n$. 817997).

\section{References}

1. Munshi NC, Anderson KC. New strategies in the treatment of multiple myeloma. Clin Cancer Res. 2013;19(13):3337-3344.

2. Barbieri M, Manzoni M, Fabris $S$, et al. Compendium of FAM46C gene mutations in plasma cell dyscrasias. Br J Haematol. 2016;174(4):642-645

3. Bolli N, Avet-Loiseau H, Wedge DC, et al. Heterogeneity of genomic evolution and mutational profiles in multiple myeloma. Nat Commun. 2014;5:2997.

4. Chapman MA, Lawrence MS, Keats JJ, et al. Initial genome sequencing and analysis of multiple myeloma. Nature. 2011; 471(7339):467-472.

5. Lionetti M, Barbieri M, Manzoni $\mathrm{M}$, et al. Molecular spectrum of TP53 mutations in plasma cell dyscrasias by next generation sequencing: an Italian cohort study and overview of the literature. Oncotarget. 2016;7(16):21353-21361.

6. Lionetti M, Barbieri M, Todoerti K, et al. A compendium of DIS3 mutations and associated transcriptional signatures in plasma cell dyscrasias. Oncotarget. 2015;6(28): 26129-26141.

7. Lohr JG, Stojanov P, Carter SL, et al Widespread genetic heterogeneity in multiple myeloma: implications for targeted therapy. Cancer Cell. 2014;25(1):91-101.

8. Bolli N, Biancon G, Moarii M, et al. Analysis of the genomic landscape of multiple myeloma highlights novel prognostic markers and disease subgroups. Leukemia. 2018;32(12):2604-2616

9. D'Agostino M, Zaccaria GM, Ziccheddu B, et al. Early relapse risk in patients with newly diagnosed multiple myeloma characterized by next-generation sequencing. Clin Cancer Res. 2020;26(18):4832-4841.

10. Walker BA, Mavrommatis K, Wardell CP, et al. A high-risk, double-hit, group of newly diagnosed myeloma identified by genomic analysis. Leukemia. 2019;33(1):159-170.

11. Tomecki R, Drazkowska K, Kucinski I, et al. Multiple myeloma-associated hDIS3 mutations cause perturbations in cellular RNA metabolism and suggest hDIS3 PIN domain as a potential drug target. Nucleic Acids Res. 2014;42(2):1270-1290
12. Schneider C, Leung E, Brown J, Tollervey D. The N-terminal PIN domain of the exosome subunit Rrp44 harbors endonuclease activity and tethers Rrp44 to the yeast core exosome. Nucleic Acids Res. 2009; 37(4): 1127-1140.

13. Lorentzen E, Basquin J, Tomecki R, Dziembowski A, Conti E. Structure of the active subunit of the yeast exosome core, Rrp44: diverse modes of substrate recruitment in the RNase II nuclease family. Mol Cell. 2008; 29(6):717-728.

14. Robinson SR, Oliver AW, Chevassut TJ, Newbury SF. The $3^{\prime}$ to 5 ' exoribonuclease DIS3: from structure and mechanisms to biological functions and role in human disease. Biomolecules. 2015;5(3):1515-1539.

15. Tomecki R, Drazkowska K, Dziembowski A. Mechanisms of RNA degradation by the eukaryotic exosome. Chembiochem. 2010; 11(7):938-945.

16. Boyle EM, Ashby C, Tytarenko RG, et al. BRAF and DIS3 mutations associate with adverse outcome in a long-term follow-up of patients with multiple myeloma. Clin Cancer Res. 2020;26(10):2422-2432. 
17. Manier S, Salem KZ, Park J, Landau DA, Getz G, Ghobrial IM. Genomic complexity of multiple myeloma and its clinical implications. Nat Rev Clin Oncol. 2017; 14(2):100-113.

18. Binder M, Rajkumar SV, Ketterling RP, et al. Prognostic implications of abnormalities of chromosome 13 and the presence of multiple cytogenetic high-risk abnormalities in newly diagnosed multiple myeloma. Blood Cancer J. 2017;7(9):e600.

19. Chiecchio L, Dagrada GP, Ibrahim AH, et al. Timing of acquisition of deletion 13 in plasma cell dyscrasias is dependent on genetic context. Haematologica. 2009; 94(12):1708-1713

20. Chesi M, Stein CK, Garbitt VM, et al. Monosomic loss of MIR15A/MIR16-1 is a driver of multiple myeloma proliferation and disease progression. Blood Cancer Discov. 2020; 1(1):68-81

21. de Groen FL, Krijgsman O, Tijssen M, et al. Gene-dosage dependent overexpression at the $13 q$ amplicon identifies DIS3 as candidate oncogene in colorectal cancer progression. Genes Chromosomes Cancer. 2014; 53(4):339-348.

22. Weissbach S, Langer C, Puppe B, et al. The molecular spectrum and clinical impact of DIS3 mutations in multiple myeloma. Br J Haematol. 2015;169(1):57-70.

23. Segalla S, Pivetti S, Todoerti K, et al. The ribonuclease DIS3 promotes let-7 miRNA maturation by degrading the pluripotency factor LIN28B mRNA. Nucleic Acids Res. 2015;43(10):5182-5193.
24. Walker BA, Mavrommatis K, Wardell CP, et al. Identification of novel mutational drivers reveals oncogene dependencies in multiple myeloma. Blood. 2018;132(6):587-597.

25. Schadt EE, Li C, Ellis B, Wong WH. Feature extraction and normalization algorithms for high-density oligonucleotide gene expression array data. J Cell Biochem Suppl. 2001;Suppl 37:120-125.

26. Subramanian A, Tamayo P, Mootha VK, et al. Gene set enrichment analysis: a knowledge-based approach for interpreting genome-wide expression profiles. Proc Natl Acad Sci U S A. 2005;102(43):1554515550.

27. Chen L, Li Q, She T, et al. IRE1alpha-XBP1 signaling pathway, a potential therapeutic target in multiple myeloma. Leuk Res. 2016;49:7-12.

28. Sigova AA, Mullen AC, Molinie B, et al Divergent transcription of long noncoding RNA/mRNA gene pairs in embryonic stem cells. Proc Natl Acad Sci U S A. 2013; 110(8):2876-2881.

29. Tan JY, Smith AAT, Ferreira da Silva M, et al. Cis-acting complex-trait-associated lincRNA expression correlates with modulation of chromosomal architecture. Cell Rep. 2017;18(9):2280-2288.

30. Trinklein ND, Aldred SF, Hartman SJ, Schroeder DI, Otillar RP, Myers RM. An abundance of bidirectional promoters in the human genome. Genome Res. 2004; 14(1):62-66.

31. Pefanis E, Basu U. RNA exosome regulates AID DNA mutator activity in the B cell genome. Adv Immunol. 2015;127:257-308.

32. Chlebowski A, Lubas $M$, Jensen $T H$ Dziembowski A. RNA decay machines: the exosome. Biochim Biophys Acta. 2013;1829(6-7):552-560.

33. Higgins LG, Hayes JD. Mechanisms of induction of cytosolic and microsomal glutathione transferase (GST) genes by xenobiotics and pro-inflammatory agents. Drug Metab Rev. 2011:43(2):92-137.

34. Xu B, Oin T, Yu J, Giordano TJ, Sartor MA, Koenig RJ. Novel role of ASH1L histone methyltransferase in anaplastic thyroid carcinoma. J Biol Chem. 2020;295(26):8834 8845.

35. Chapman J, Ng YS, Nicholls TJ. The maintenance of mitochondrial DNA integrity and dynamics by mitochondrial membranes. Life. 2020;10(9):164.

36. Davidson L, Francis L, Cordiner RA, et al. Rapid depletion of DIS3, EXOSC10, or XRN2 reveals the immediate impact of exoribonucleolysis on nuclear RNA metabolism and transcriptional control. Cell Rep. 2019;26(10):2779-2791.e5.

37. Kiss DL, Andrulis ED. Genome-wide analysis reveals distinct substrate specificities of Rrp6, Dis3, and core exosome subunits. RNA. 2010;16(4):781-791

38. Milbury KL, Paul B, Lari A, et al Exonuclease domain mutants of yeast DIS3 display genome instability. Nucleus. 2019;10(1):21-32.

39. Rigby RE, Rehwinkel J. RNA degradation in antiviral immunity and autoimmunity. Trends Immunol. 2015;36(3):179-188. 